\title{
Saccharomyces cerevisiae Hrq1 helicase activity is affected by the sequence but not the length of single-stranded DNA
}

\author{
Cody M. Rogers, Matthew L. Bochman* \\ Molecular and Cellular Biochemistry Department, 212 South Hawthorne Drive, Simon Hall MSB1, Room 405B, Indiana University, Bloomington, IN 47405, \\ USA
}

\section{A R T I C L E I N F O}

Article history:

Received 27 March 2017

Accepted 1 April 2017

Available online $\mathrm{xxx}$

\section{Keywords:}

DNA helicase

RecQ

Hrq1

RecQ4

Saccharomyces cerevisiae

\begin{abstract}
A B S T R A C T
Mutations in the human RecQ4 DNA helicase are associated with three different diseases characterized by genomic instability. To gain insight into how RecQ4 dysfunction leads to these pathologies, several groups have used the Saccharomyces cerevisiae RecQ4 homolog Hrq1 as an experimental model. Hrq1 displays many of the same functions as RecQ4 in vivo and in vitro. However, there is some disagreement in the literature about the effects of single-stranded DNA (ssDNA) length on Hrq1 helicase activity and the ability of Hrq1 to anneal complementary ssDNA oligonucleotides into duplex DNA. Here, we present a side-by-side comparison of Hrq1 and RecQ4 helicase activity, demonstrating that in both cases, long random-sequence $3^{\prime}$ ssDNA tails inhibit DNA unwinding in vitro in a length-dependent manner. This appears to be due to the formation of secondary structures in the random-sequence ssDNA because Hrq1 preferentially unwound poly(dT)-tailed forks independent of ssDNA length. Further, RecQ4 is capable of ssDNA strand annealing and annealing-dependent strand exchange, but Hrq1 lacks these activities. These results establish the importance of DNA sequence in Hrq1 helicase activity, and the absence of Hrq1 strand annealing activity explains the previously identified discrepancies between S. cerevisiae Hrq1 and human RecQ4.
\end{abstract}

(C) 2017 Elsevier Inc. All rights reserved.

\section{Introduction}

The disease-linked RecQ family helicases are evolutionarily conserved from a single RecQ in bacteria to five RecQs in humans (RecQ1, BLM, WRN, RecQ4, and RecQ5) [1]. Mutations in three RecQs (BLM, WRN, and RecQ4) are directly implicated in human diseases characterized by genomic instability. Unlike BLM and WRN, it is currently unclear how RecQ4 contributes to maintaining genome stability. This is largely due to technical challenges of working with RecQ4 both in vivo and in vitro. For instance, the presence of a Sld2-like domain (an essential replication initiation factor in yeast) at the $N$-terminus of RecQ4 makes mutational analysis difficult as mutations could disrupt DNA replication [2], which is independent of RecQ4-mediated DNA repair [3]. The Sld2like domain also confounds biochemical analysis because

Abbreviations: ssDNA, single-stranded DNA; dsDNA, double-stranded DNA; ICL, inter-strand crosslink; EMSA, electrophoretic mobility shift assay.

* Corresponding author.

E-mail addresses: codroger@indiana.edu (C.M. Rogers), bochman@indiana.edu (M.L. Bochman).
Saccharomyces cerevisiae Sld2 is a known annealase [4], and the Sld2-like portion of RecQ4 plays a significant role in DNA annealing activity [5]. This ability of RecQ4 to anneal complementary DNA substrates is so robust that it initially masked the DNA unwinding activity of the enzyme [6], making RecQ4 helicase activity difficult to analyze. Thus, a model for study of RecQ4 that circumvents these technical challenges was needed.

In-depth bioinformatics revealed Saccharomyces cerevisiae Hrq1 as a homolog of RecQ4 [7]. Hrq1 lacks the Sld2-like domain found in metazoan RecQ4 sub-family helicases, alleviating the issue of replication confounding the DNA repair function of the helicase. Furthermore, Hrq1 over-expression and purification produces abundant amounts of protein relative to RecQ4 [8]. Since its discovery, Hrq1 has been determined to play important roles at telomeres such as maintaining telomere length homeostasis in the absence of the known telomerase inhibitor Pif1 [9]. Additionally, Hrq1 functions in the repair of DNA inter-strand crosslinks (ICL) in the same pathway as the ICL repair nuclease Pso2 [8,9]. Telomere maintenance and ICL repair are likewise associated with human RecQ4 $[10,11]$. In vitro, Hrq1 and RecQ4 helicase activity is stimulated by poly(dT) and telomeric repeat ssDNA [8]. Both helicases preferentially bind to Holliday junctions and D-Loop DNA 
structures, with the latter being a preferred substrate for unwinding. Finally, 2D averaging of each helicase via negative staining and transmission electron microscopy shows that both helicases adopt similar tertiary structures, which are distinct from RecQ1.

Concerning DNA unwinding by Hrq1, a weak in vitro helicase activity has been reported for the $S$. pombe Hrq1 helicase [12], but the activity displayed by $S$. cerevisiae Hrq1 is more robust $[8,9,13]$. However, there is a discrepancy in the $S$. cerevisiae Hrq1 literature. Using recombinant Hrq1 over-expressed and purified in two different ways, it has been found that Hrq1 can unwind model fork substrates with ssDNA tails of $25 \mathrm{nt}$ in length. In contrast, another group reports an "absolute requirement for a long 3'-tail ( $\geq 70 \mathrm{nt})$ for efficient unwinding" by Hrq1 [13]. Similarly, it is unclear if Hrq1 lacks single-stranded DNA (ssDNA) annealing activity $[8,9]$ or if it is an annealase like human RecQ4 [13]. The current dearth of Hrq1 literature amplifies these discrepancies and complicates follow-up research as proper substrate design is pivotal for accurately reconstituting biochemical pathways in vitro.

Here, we compared the effects of ssDNA tail length and sequence on Hrq1 helicase activity, as well as assayed for annealing activity. We found that increasing the length of the randomsequence ssDNA tails on fork substrates inhibited Hrq1 binding and unwinding, likely due to the higher propensity for longer stretches of ssDNA to form secondary structures. Using equivalent forks with poly(dT) ssDNA tails eliminated length-induced inhibition of Hrq1 helicase activity. However, Hrq1 unwinding of forks with poly(dT)-tails of 25-, 50-, and 75-nt in length was indistinguishable, indicating that long ( $\geq 70 \mathrm{nt}$ ) 3'-tails are not required for robust helicase activity. Finally, we demonstrated that the strong DNA strand annealing and exchange activities previously reported for RecQ4 [5] are absent in Hrq1.

\section{Materials and methods}

\subsection{Nucleotides, oligonucleotides, and other reagents}

${ }^{32} \mathrm{P}$-ATP was purchased from PerkinElmer (Waltham, MA), and unlabeled ATP was from GE Healthcare (Little Chalfont, UK) or DOT Scientific (Burton, MI). The oligonucleotides used in this work were synthesized by IDT (Coralville, IA) and are listed in Supplementary Table 1. All restriction enzymes were from New England Biolabs (Ipswich, MA).

\subsection{Protein purification}

S. cerevisiae Hrq1 and human RecQ4 were purified as described [8]. Briefly, the proteins were over-expressed in baculovirusinfected insect cells and purified by nickel affinity (Hrq1) or using amylose resin and Strep-Tactin Superflow (IBA) chromatography. Purification was analyzed by SDS-PAGE and Coomassie staining. Peak fractions were pooled and dialyzed against storage buffer (25 mM Na-HEPES [pH 8], 30\% glycerol, $300 \mathrm{mM} \mathrm{NaOAc} \mathrm{[pH} \mathrm{7.6],}$ $25 \mathrm{mM} \mathrm{NaCl}, 5 \mathrm{mM}$ MgOAc, $1 \mathrm{mM}$ DTT, and 0.1\% Tween-20). Protein concentrations were quantified on SYPRO orange (Sigma) stained SDS-PAGE gels using BSA dilutions to plot a standard curve. Gel imaging and analysis were performed using a Typhoon 9210 Variable Mode Imager (Amersham Biosciences).

\subsection{DNA substrates}

Substrates were 5'-end labelling with T4 polynucleotide kinase (T4 PNK; NEB) and $\gamma-\left[{ }^{32} \mathrm{P}\right]-\mathrm{ATP}$. Labelled oligonucleotides were separated from free label using illustra ProbeQuant G-50 micro columns (GE Healthcare) following the manufacturer's instructions. Oligonucleotides were annealed by incubating complementary or partially complementary oligonucleotides overnight at $37{ }^{\circ} \mathrm{C}$ in Annealing Buffer (20 mM Tris- $\mathrm{HCl}$ [pH 8], 4\% glycerol, 0.1 mM EDTA, $40 \mu \mathrm{g} / \mathrm{mL}$ BSA, $10 \mathrm{mM}$ DTT, and $10 \mathrm{mM} \mathrm{MgOAc}$ ) [4]. The sequences of the oligonucleotides used to make DNA substrates are listed in Supplementary Table 1.

\subsection{DNA binding}

Electrophoretic mobility shift assays (EMSAs) were performed for $30 \mathrm{~min}$ at $30^{\circ} \mathrm{C}$ in $1 \times$ binding buffer ( $25 \mathrm{mM}$ Na-HEPES [pH 8.0], $5 \%$ glycerol, $50 \mathrm{mM}$ NaOAc [pH 7.6], $150 \mu \mathrm{M} \mathrm{NaCl}, 7.5 \mathrm{mM} \mathrm{MgOAc}$, and $0.01 \%$ Tween-20) with $0.1 \mathrm{nM}$ radiolabeled fork substrate. Protein-DNA complexes were separated on 8\% 19:1 acrylamide:bisacrylamide gels at $10 \mathrm{~V} / \mathrm{cm}$ in TBE $(90 \mathrm{mM}$ Tris- $\mathrm{HCl}$ [pH 8.0], $90 \mathrm{mM}$ boric acid, and $2 \mathrm{mM}$ EDTA [pH 8.0]). Gels were dried under vacuum and imaged using a Typhoon 9210 Variable Mode Imager. DNA binding was quantified using ImageQuant 5.2 software.

\subsection{Helicase assay}

DNA unwinding was assessed by incubating the indicated concentrations of helicase with $5 \mathrm{mM}$ ATP, $0.1 \mathrm{nM}$ radiolabelled fork, and $1 \times$ unwinding buffer ( $25 \mathrm{mM}$ Na-HEPES [pH 8.0], 5\% glycerol, $50 \mathrm{mM}$ NaOAc [pH 7.6], $150 \mu \mathrm{M} \mathrm{NaCl}, 7.5 \mathrm{mM} \mathrm{MgOAc}$, and $0.01 \%$ Tween-20). RecQ4 helicase assays were performed in the presence of $15 \mathrm{nM}$ cold ssDNA trap [14]. ATP was required to observe unwinding, suggesting that strand exchange was not the activity being measured (data not shown) [5]. Reactions were incubated at $37^{\circ} \mathrm{C}$ for $30 \mathrm{~min}$ and stopped with the addition of $1 \times$ Stop-Load dye (5\% glycerol, $20 \mathrm{mM}$ EDTA, 0.05\% SDS, and 0.25\% bromophenol blue) supplemented with $400 \mu \mathrm{g} / \mathrm{mL}$ SDS-Proteinase K followed by a 1-min incubation at $37{ }^{\circ} \mathrm{C}$. Unwound DNA was separated on $8 \%$ 19:1 acrylamide:bis-acrylamide gels in TBE buffer at $10 \mathrm{~V} / \mathrm{cm}$ and imaged as for the DNA binding assay.

\subsection{DNA annealing and strand exchange assays}

Annealing reactions were performed using $0.1 \mathrm{nM}$ each of the partially complementary oligonucleotides MB1057 and MB1059 or MB1167 and MB1168 (Supplementary Table 1) that can be annealed to form a fork. These oligonucleotides were incubated with no protein, Hrq1, or RecQ4 for the indicated times at $37^{\circ} \mathrm{C}$ in $1 \times$ unwinding buffer. Reactions were stopped by the addition of $1 \times$ StopLoad dye supplemented with $400 \mu \mathrm{g} / \mathrm{mL}$ SDS-Proteinase $\mathrm{K}$ and incubated at $37{ }^{\circ} \mathrm{C}$ for $1 \mathrm{~min}$. Reactions were then loaded onto $10 \%$ 19:1 acrylamide:bis-acrylamide gels, run at $10 \mathrm{~V} / \mathrm{cm}$ in TBE buffer, and imaged as above.

Strand annealing-dependent strand exchange assays used $0.1 \mathrm{nM}$ fork (the annealed product of oligonucleotides MB1057 and MB1059) incubated with $0.1 \mathrm{nM}$ of oligonucleotide MB1058, which is fully complementary to MB1057 such that strand exchange results in conversion of the forked structure into a faster-migrating blunt ended double-stranded DNA (dsDNA) substrate. These assays were performed in the same conditions as for annealing activity, and products were separated on 12\% 19:1 acrylamide:bisacrylamide gels in TBE buffer at $10 \mathrm{~V} / \mathrm{cm}$ and imaged as above. The fork oligonucleotides used in the strand annealing and strand exchange assays were identical to those used previously to identify RecQ4-mediated strand exchange activity [5].

\subsection{Statistical analyses}

All data were analyzed and plotted using GraphPad Prism 6 (GraphPad Software, Inc). The plotted values are averages, and the error bars were calculated as the standard deviation from three or 
more independent experiments. For binding and unwinding curves, $p$-values were determined by the extra sum-of-squares $\mathrm{F}$ test. The $p$-values for annealing were determined using an unpaired $t$-test. We defined statistical significance as $p<0.01$.

\section{Results}

\subsection{Long ssDNA tails are not required to stimulate Hrq1 helicase activity}

It has been reported that a long ( $\geq 70 \mathrm{nt}$ ) 3'-ssDNA tail is "absolutely required" to stimulate duplex unwinding by Hrq1 [13]. This is in disagreement with our previous work demonstrating that the same concentration of $S$. cerevisiae $\mathrm{Hrq} 1(50 \mathrm{nM})$ unwinds a fork substrate with only 25-nt ssDNA tails [8,9]. However, Hrq1 was generated in insect cells by baculovirus over-expression in Ref. [13], and we previously over-expressed our Hrq1 in E. coli [9]. Thus, differences in the over-expression host (e.g., differential posttranslational modification (PTM)) may underlie this discrepancy. Because we recently optimized Hrq1 purification using baculovirus-mediated over-expression in insect cells [8], we revisited this topic with our current Hrq1 preparations.

First, comparing Hrq1 binding to forks containing 25-, 50-, and 75-nt random-sequence ssDNA tails, we found that increasing ssDNA tail length inhibited binding by Hrq1 in a length-dependent manner (Fig. 1A and Table 1). Similarly, when measuring the DNA unwinding of these same substrates (Fig. 1B), increasing ssDNA tail length also inhibited the helicase activity of Hrq1 (Table 1). Therefore, regardless of over-expression in E. coli or insect cell culture, S. cerevisiae Hrq1 displayed robust helicase activity on DNA forks with short (25-nt) ssDNA tails, and longer tracts of ssDNA were inhibitory.

We also performed the same analyses with RecQ4 to determine if this is an evolutionarily conserved phenomenon among RecQ4 sub-family helicases. In contrast to Hrq1, the EMSAs revealed that increasing ssDNA tail length increased RecQ4 binding affinity for the fork (Fig. 1C and Table 1). However, the effects of ssDNA tail length on RecQ4 helicase activity mirrored those of Hrq1. Substrates with longer tails were poorly unwound by RecQ4 (Fig. 1D and Table 1). These results are discussed in relation to [13] below.

\subsection{Hrq1 preferentially unwinds substrates with poly(dT) ssDNA tails}

We previously reported that Hrq1 vigorously unwinds poly(dT)tailed forks [8]. When comparing the migration through native PAGE gels of radiolabeled DNA forks containing random-sequence ssDNA tails with forks containing poly(dT) ssDNA tails, we noticed that the forks with random-sequence tails migrated more quickly than the equivalently sized poly(dT)-tailed forks (Fig. 2A). This was likely due to the random-sequence ssDNA forming more compact secondary structures; under our assay conditions, pol$y(d T)$ tracts are unable to form secondary structures $[15,16]$. To determine if the inhibition of Hrq1 binding and unwinding longer forks is due to secondary structure formation in the randomsequence ssDNA tails, we measured Hrq1 binding and unwinding on forks with 25-, 50-, and 75-nt poly(dT) tails. Contrary to Hrq1 binding to forks with random-sequence tails, the longer 50- and 75 -nt poly(dT) tails were bound slightly better than the 25-nt poly(dT)-tailed fork $(p<0.0001)$ (Fig. 2 B and Table 1$)$. However, Hrq1 unwound the poly $(\mathrm{dT})$ forks independently of ssDNA length as the $K_{M}$ for each substrate was not significantly different ( $p=0.4009$ ) (Fig. 2C and Table 1$)$. Thus, the ability of the longer random-sequence tails to form secondary structure likely inhibited Hrq1 binding and unwinding of these substrates. Furthermore,
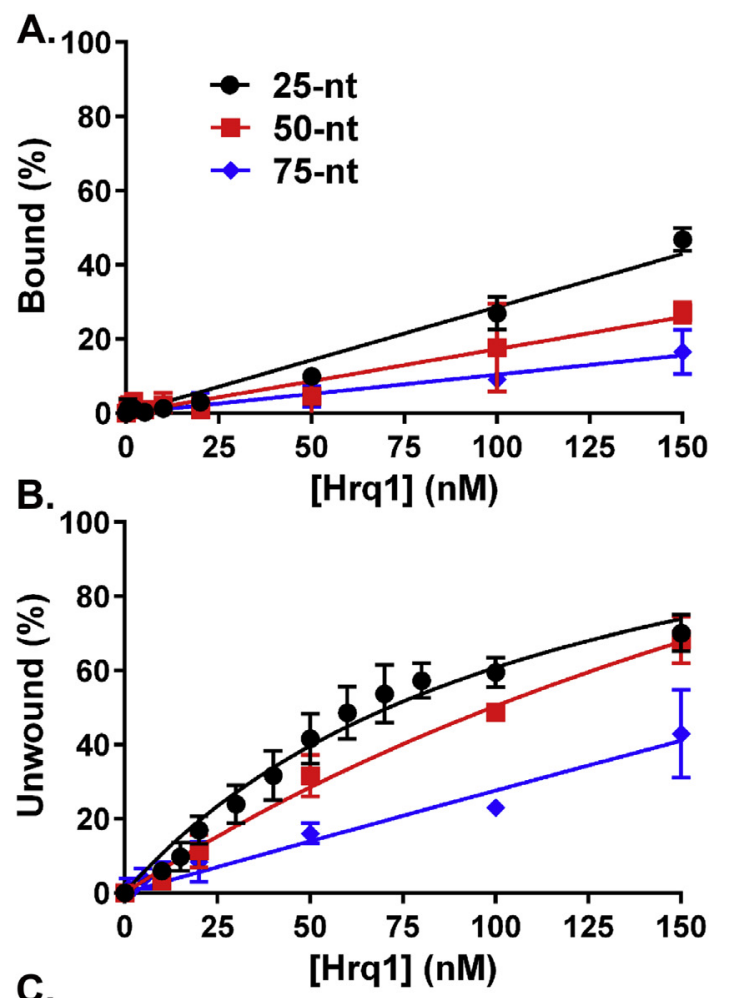

C
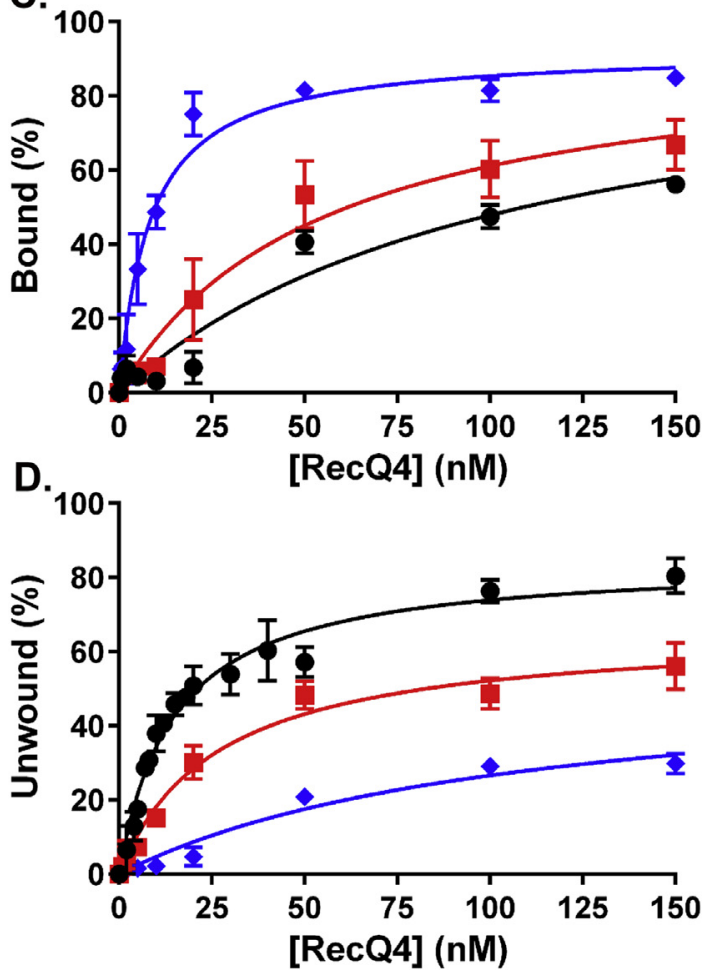

Fig. 1. Long random-sequence ssDNA tails decrease helicase activity. A) Hrq1 binding to DNA forks with 25-, 50-, or 75-nt random-sequence tails decreases as tail length increases. B) Hrq1 unwinding of DNA forks with 25-, 50-, or 75-nt random-sequence tails decreases as tail length increases. C) RecQ4 binding to DNA forks with 25-, 50-, or 75-nt random-sequence tails increases as tail length increases. D) RecQ4 unwinding of DNA forks with 25-, 50-, or 75-nt random-sequence tails decreases as tail length increases.

Hrq1 activity is largely dictated by DNA sequence as observed by comparing the $K_{\mathrm{d}}$ and $K_{M}$ values between random-sequence and poly(dT)-tailed forks of the same length (Table 1). 
Table 1

Dissociation constants $\left(K_{\mathrm{d}}\right)$ and apparent Michaelis constants $\left(K_{M}\right)$ for Hrq1 and RecQ4.

\begin{tabular}{|c|c|c|c|c|}
\hline \multirow[t]{2}{*}{ Fork tail sequence } & \multicolumn{2}{|l|}{ Hrq1 } & \multicolumn{2}{|l|}{ RecQ4 } \\
\hline & Binding $K_{\mathrm{d}}(\mathrm{nM})$ & Unwinding $K_{M}(\mathrm{nM})$ & Binding $K_{\mathrm{d}}(\mathrm{nM})$ & Unwinding $K_{M}(\mathrm{nM})$ \\
\hline 25-nt Random ${ }^{a}$ & $\sim 150$ & $113.9 \pm 19.5$ & $107.7 \pm 32.5$ & $14.7 \pm 1.1$ \\
\hline 50-nt Random & $>150$ & $331.8 \pm 125.4$ & $52.4 \pm 12.6$ & $26.4 \pm 3.7$ \\
\hline 75-nt Random & $>150$ & 11420 & $8.3 \pm 1.0$ & $106.7 \pm 30.0$ \\
\hline 25-nt poly(dT) & $11.3 \pm 2.2$ & $5.3 \pm 0.9$ & $\mathrm{~N} / \mathrm{D}$ & $\mathrm{N} / \mathrm{D}$ \\
\hline 50-nt poly(dT) & $2.1 \pm 0.3$ & $4.8 \pm 0.5$ & $\mathrm{~N} / \mathrm{D}$ & $\mathrm{N} / \mathrm{D}$ \\
\hline 75-nt poly (dT) & $1.2 \pm 0.2$ & $6.1 \pm 0.8$ & $\mathrm{~N} / \mathrm{D}$ & $\mathrm{N} / \mathrm{D}$ \\
\hline
\end{tabular}

a Binding and unwinding constants for the 25-nt Random Fork come from Ref. [8].

A.

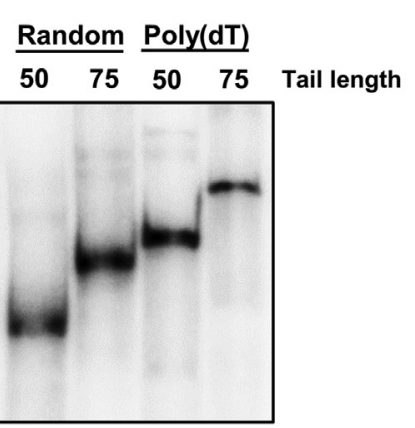

B.

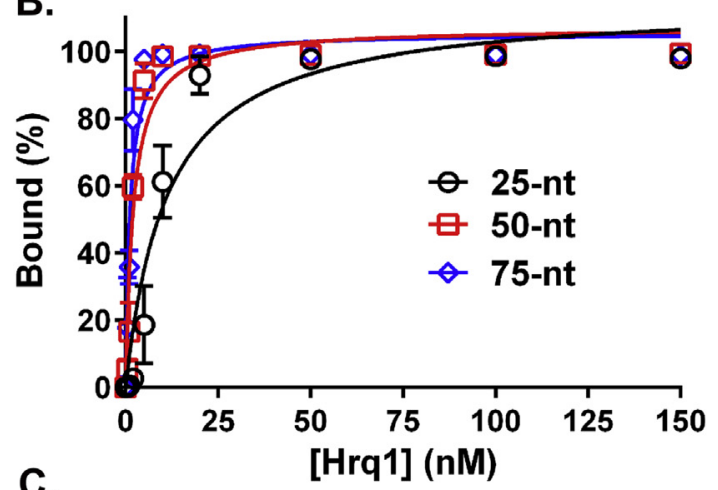

C.

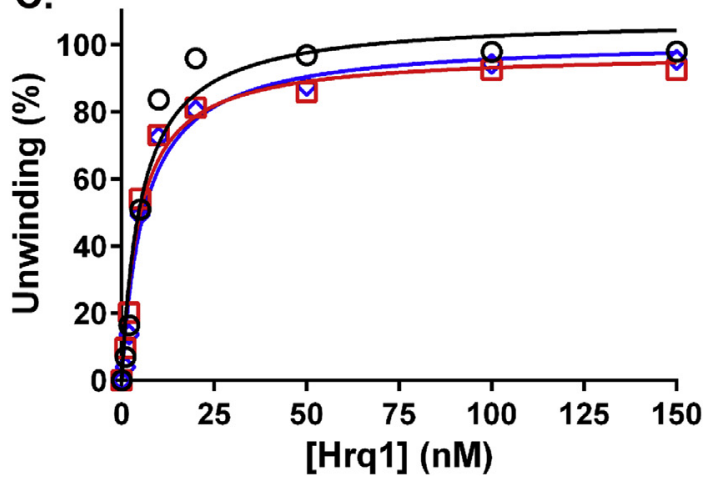

Fig. 2. Secondary structure formation inhibits Hrq1 DNA binding and unwinding. A) Differential mobility through a native PAGE gel of DNA forks containing 50- and 75-nt ssDNA tails comprised of random or poly $(\mathrm{dT})$ sequence. The faster migration of the random-sequence forks suggests secondary structure formation. B) Increasing the poly $(\mathrm{dT})$ tail length has little-to-no effect on Hrq1 binding affinity for these fork substrates. C) Increasing the poly(dT) tail length has no effect on Hrq1 unwinding activity for these fork substrates.

\subsection{Hrq1 does not possess ssDNA annealing activity}

Measuring RecQ4 helicase activity in vitro has historically been difficult due to its strong strand annealing activity, which masks
DNA unwinding in the absence of cold DNA trap [14]. Strand annealing activity is attributed to the Sld2-like $\mathrm{N}$-terminal domain of RecQ4 [5,17], which is further supported by the demonstrated DNA annealing activity of S. cerevisiae Sld2 [4]. We previously found no hint of DNA strand annealing by Hrq1 [8], presumably because it does not contain a Sld2-like domain. However, others report a "potent DNA strand annealing activity" for their recombinant Hrq1 preparations [13]. As above, this discrepancy could have been due to lack of activating PTMs from E. coli over-expression compared to generation of Hrq1 in baculovirus-infected insect cells, so we again assayed for Hrq1 annealing activity using our new recombinant Hrq1 prepared from insect cells.

To determine if Hrq1 is capable of annealing complementary ssDNA sequences, we performed side-by-side strand annealing assays with $20 \mathrm{nM}$ Hrq1 and RecQ4 by incubating each enzyme with two partially complementary oligonucleotides that can be annealed in vitro to form a fork. Under these conditions, spontaneous annealing in the absence of protein occurs at a low rate ( $10.1 \pm 4.7 \%$ by $10 \mathrm{~min}$ ), which is catalyzed by the addition of RecQ4. Indeed, annealed product appeared above background levels in as quickly as $1 \mathrm{~min}$ in the presence of RecQ4, and by $10 \mathrm{~min}$, RecQ4 annealed $>70 \%$ of the ssDNA (Fig. 3A). This is consistent with the strong RecQ4 annealing that has been reported previously [5]. In contrast, the addition of Hrq1 had no effect relative to the noprotein control $(8.3 \pm 4.7 \%$ at $10 \mathrm{~min} ; p=0.6604)$. As shown previously, increasing protein concentration to $100 \mathrm{nM}$ and increasing reaction time to 30 min results in RecQ4 annealing nearly $100 \%$ of the ssDNA, but Hrq1 still does not display any annealing activity above background under these conditions [8]. Further, neither using the higher affinity 25-nt tailed poly(dT) fork nor the exact in vitro annealing conditions reported in Ref. [13] had any effect on the inability of Hrq1 to anneal ssDNA (Fig. 3B and data not shown). This inability of Hrq1 to anneal DNA is further supported by the absence of any effect of cold trap DNA on apparent unwinding activity (data not shown and $[8,9]$ ).

\subsection{Hrq1 does not possess strand exchange activity}

The BLM and WRN helicases are capable of ssDNA strand exchange [18], which is measured by incubating a duplexed or partially duplexed DNA substrate with a complementary oligonucleotide such that the swapping of one of the duplex strands with the ssDNA can be measured by differential mobility on a native gel. This activity was also recently identified to be associated with the $\mathrm{N}$-terminus of RecQ4 [5]. Using identical fork substrates to Keller et al. (2014), we assayed for strand exchange by Hrq1 and RecQ4. Similar to previous results, we observed strand exchange activity in the presence of $50 \mathrm{nM}$ RecQ4, but an equivalent amount of Hrq1 was unable to produce the strand exchange product (Fig. 3C). When increasing the helicase concentration to $100 \mathrm{nM}$, we were still unable to detect any strand exchange product for Hrq1 above the no-protein control levels (data not shown). The inability of Hrq1 to 

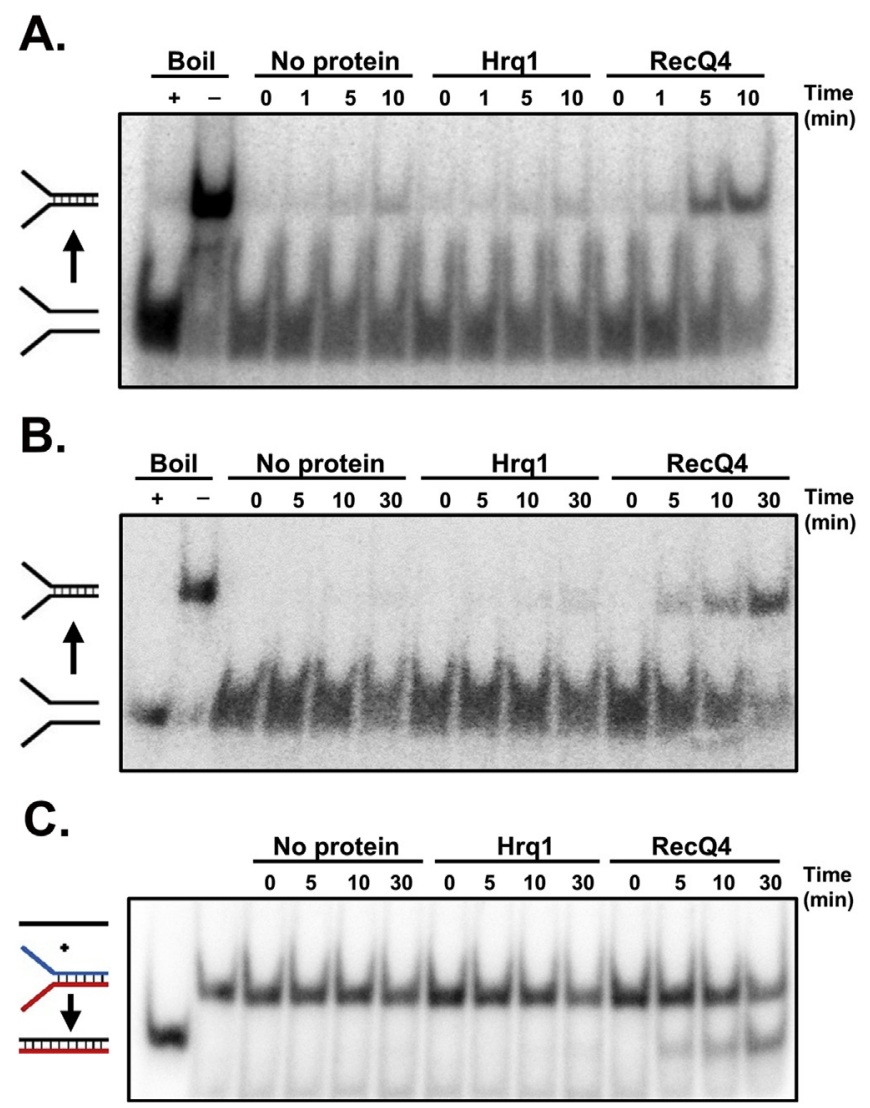

Fig. 3. Hrq1 lacks DNA strand annealing and strand exchange activities. A) DNA strand annealing in the absence of protein or presence of $20 \mathrm{nM}$ Hrq1 or RecQ4. In this reaction, two ssDNAs (one radiolabeled) can be annealed to form a fork. The "Boil +" lane indicates the position at which the un-annealed radiolabeled oligonucleotide migrates in the gel; the "Boil -“ lane indicates the position at which the annealed product migrates in the gel. By 10 min, RecQ 4 annealed $>70 \%$ of the substrate ssDNAs, but Hrq1 did not catalyze annealing above background levels. B) Similar assay to that in A) but using the preferred poly(dT) fork oligonucleotides, $100 \mathrm{nM}$ protein, and a longer time course. C) DNA strand exchange in the absence of protein or presence of $50 \mathrm{nM} \mathrm{Hrq1}$ or RecQ4. In this reaction, the unlabeled partially complementary oligonucleotide that forms half of the fork substrate in A) can be exchanged for a completely complementary unlabeled oligonucleotide to produce blunt dsDNA, which migrates more quickly in the gel than the fork. RecQ4 strand exchange activity is evident by 5 min and reaches $\sim 50 \%$ by 30 min, but Hrq1 did not catalyze strand exchange above background levels.

perform strand exchange is likely due to its lack of strand annealing activity (Fig. 3A and B), which is postulated to be required for strand exchange [5]. We hypothesize that the difference in strand annealing/exchange activity between Hrq1 and RecQ4 is due to the absence of the Sld2-like domain in Hrq1.

\section{Discussion}

It has been reported that recombinant $S$. cerevisiae Hrq1 requires $\geq 70 \mathrm{nt}$ of $3^{\prime}$ ssDNA for efficient helicase activity in vitro and that it displays DNA strand annealing activity [13]. This disagrees with our previous work on Hrq1 demonstrating in vitro helicase activity on substrates with ssDNA tails as short as 25-nt tails [8,9] and our unpublished observations that recombinant Hrq1 cannot anneal complementary ssDNA oligonucleotides into dsDNA. Our former Hrq1 preparations were generated by over-expression in E. coli, while those of Kwon et al. (2012) were produced in baculovirusinfected insect cells [13]. As a eukaryotic expression host, generating recombinant eukaryotic proteins in Spodoptera frugiperda cells aids in protein folding and maturation, and it can result in more native-like PTMs [19]. Thus, it was possible that our Hrq1 produced in E. coli was not folded into a native conformation and/or lacked PTMs, affecting helicase activity or inactivating annealing activity.

To address this issue, we developed an optimized overexpression and purification protocol for Hrq1 that includes baculovirus and insect cell culture [8], similar to previous reports [13]. However, with recombinant Hrq1 generated in this manner, we still observed vigorous helicase activity on substrates with short (25-nt) ssDNA tails (Figs. 1B and 2C, and Table 1) [8]. Further, increasing the length of the poly(dT) ssDNA tails on our forks had no effect on unwinding activity, but increasing the random-sequence ssDNA tail length on the fork substrates actually decreased DNA unwinding by Hrq1 (Fig. 1B). The latter phenomenon was likely due to secondary structure formation by the random-sequence ssDNA (Fig. 2A). It may also be due to Hrq1's lower binding affinity for forks with longer random-sequence tails (Fig. $1 \mathrm{~A}$ and Table 1 ), inherent poor processivity during ssDNA translocation toward the duplex to be unwound, or a combination thereof. Regardless, this inhibition of Hrq1 helicase activity by long random-sequence ssDNA tracts was also displayed by RecQ4 on the same fork substrates (Fig. 1D), indicating that it is a property of RecQ4 sub-family helicases that is conserved between yeast and humans.

Our previous work clearly demonstrates that DNA sequence and structure play a significant role in Hrq1 and RecQ4 helicase activity [8]. Importantly, poly(dT)-tailed forks were significantly bound and unwound at levels above the random-sequence tail equivalents. In this study, Hrq1 was again more active on poly(dT) forks than forks with random-sequence ssDNA tails, but the activity on poly(dT) forks was independent of the tail length (Fig. 2B). We conclude that Hrq1 does not require a long (i.e., $\geq 70$ nt) 3'-tail for effective helicase activity as previously reported [13]. Instead, based on this and previous work [8], we propose that DNA sequence and structure preferences are more important and distinguishing characteristics for RecQ4 sub-family helicase substrates. Thus, caution should be used when designing and comparing biochemical activities between substrates for these enzymes.

Using our new recombinant Hrq1 preparations, we again failed to observe DNA strand annealing activity for Hrq1 (Fig. 3), even when using a preferred poly(dT) fork substrate [13]. In contrast, the annealing activity of RecQ4 under identical conditions was evident and strong (Fig. 3). We hypothesize that RecQ4 displays annealing activity due to the presence of the Sld2-like domain in its $\mathrm{N}$-terminus, because $S$. cerevisiae Sld2 is an in vitro annealase [20]. Hrq1 lacks a homologous domain, and therefore, it is consistent that it also lacks DNA annealing activity. As strand exchange by RecQ4 is thought to be dependent on DNA annealing activity, it is also not surprising that Hrq1 lacks strand exchange activity (Fig. 3C).

Based on our careful side-by-side biochemical comparison of Hrq1 and RecQ4 (above and [8]), as well as MS analysis of our recombinant helicase preparations demonstrating their purity [8], we are confident that the in vitro activities we assayed are due to Hrq1 and RecQ4 themselves and not to a contaminant from insect cells. In contrast, the Hrq1 preparations from Ref. [13] that required long ssDNA tails for unwinding and displayed annealing activity may have contained contaminating proteins with DNA helicase and annealing activity, leading to the discrepancies in our biochemical results. The analyses above, in conjunction with the recent and extensive in vitro characterization of Hrq1 and RecQ4 [8], establish a more reasonable ( 25-nt) 3'-tail length for efficient helicase activity and identify structures that allude to the in vivo functions of these helicases, including roles in telomere maintenance and DNA recombination. Thus, these data will be important in providing additional avenues for delineating the role of RecQ4 sub-family helicases in maintaining genome stability. 


\section{Conflict of interest}

The authors declare that no conflict of interest exists.

\section{Acknowledgements}

We thank members of the Bochman Lab for providing comments on the experiments and manuscript. This work was supported by funds from the College of Arts and Sciences, Indiana University, the Indiana University Collaborative Research Grant fund of the Office of the Vice President for Research, and the American Cancer Society [RSG-16-180-01-DMC].

\section{Appendix A. Supplementary data}

Supplementary data related to this article can be found at http:// dx.doi.org/10.1016/j.bbrc.2017.04.003.

\section{References}

[1] W.K. Chu, I.D. Hickson, RecQ helicases: multifunctional genome caretakers, Nature reviews, Cancer 9 (2009) 644-654.

[2] Y. Liu, Rothmund-Thomson syndrome helicase, RECQ4: on the crossroad between DNA replication and repair, DNA Repair 9 (2010) 325-330.

[3] L. Larizza, G. Roversi, L. Volpi, Rothmund-Thomson syndrome, Orphanet J. Rare Dis. 5 (2010) 2.

[4] D.M. Kanter, D.L. Kaplan, Sld2 binds to origin single-stranded DNA and stimulates DNA annealing, Nucleic Acids Res. 39 (2011) 2580-2592.

[5] H. Keller, K. Kiosze, J. Sachsenweger, S. Haumann, O. Ohlenschlager, T. Nuutinen, J.E. Syvaoja, M. Gorlach, F. Grosse, H. Pospiech, The intrinsically disordered amino-terminal region of human RecQL4: multiple DNA-binding domains confer annealing, strand exchange and G4 DNA binding, Nucleic Acids Res. 42 (2014) 12614-12627.

[6] M.A. Macris, L. Krejci, W. Bussen, A. Shimamoto, P. Sung, Biochemical characterization of the RECQ4 protein, mutated in Rothmund-Thomson syndrome,
DNA Repair (Amst) 5 (2006) 172-180.

[7] F. Barea, S. Tessaro, D. Bonatto, In silico analyses of a new group of fungal and plant RecQ4-homologous proteins, Comput. Biol. Chem. 32 (2008) 349-358.

[8] C.M. Rogers, J.C.-Y. Wang, H. Noguchi, T. Imasaki, Y. Takagi, M.L. Bochman, Yeast Hrq1 shares structural and functional homology with the disease-linked human RecQ4 helicase, Nucleic Acids Res. (2017). https://academic.oup.com/ nar/article/3052768/Yeast-Hrq1-shares-structural-and-functional.

[9] M.L. Bochman, K. Paeschke, A. Chan, V.A. Zakian, Hrq1, a homolog of the human RecO4 helicase, acts catalytically and structurally to promote genome integrity, Cell Rep. 6 (2014) 346-356.

[10] W. Jin, H. Liu, Y. Zhang, S.K. Otta, S.E. Plon, L.L. Wang, Sensitivity of RECQL4deficient fibroblasts from Rothmund-Thomson syndrome patients to genotoxic agents, Hum. Genet. 123 (2008) 643-653.

[11] A.K. Ghosh, M.L. Rossi, D.K. Singh, C. Dunn, M. Ramamoorthy, D.L. Croteau, Y. Liu, V.A. Bohr, RECQL4, the protein mutated in Rothmund-Thomson syndrome, functions in telomere maintenance, J. Biol. Chem. 287 (2012) 196-209.

[12] L.M. Groocock, J. Prudden, J.J. Perry, M.N. Boddy, The RecQ4 orthologue Hrq1 is critical for DNA interstrand cross-link repair and genome stability in fission yeast, Mol. Cell Biol. 32 (2012) 276-287.

[13] S.H. Kwon, D.H. Choi, R. Lee, S.H. Bae, Saccharomyces cerevisiae Hrq1 requires a long 3'-tailed DNA substrate for helicase activity, Biochem. Biophys. Res. Commun. 427 (2012) 623-628.

[14] X. Xu, Y. Liu, Dual DNA unwinding activities of the Rothmund-Thomson syndrome protein, RECQ4, EMBO J. 28 (2009) 568-577.

[15] J.B. Mills, E. Vacano, P.J. Hagerman, Flexibility of single-stranded DNA: use of gapped duplex helices to determine the persistence lengths of poly(dT) and poly(dA), J. Mol. Biol. 285 (1999) 245-257.

[16] R. Bar-Ziv, A. Libchaber, Effects of DNA sequence and structure on binding of RecA to single-stranded DNA, Proc. Natl. Acad. Sci. U. S. A. 98 (2001) 9068-9073.

[17] H. Sedlackova, B. Cechova, J. Mlcouskova, L. Krejci, RECQ4 selectively recognizes Holliday junctions, DNA repair 30 (2015) 80-89.

[18] A. Machwe, L. Xiao, J. Groden, S.W. Matson, D.K. Orren, RecQ family members combine strand pairing and unwinding activities to catalyze strand exchange, J. Biol. Chem. 280 (2005) 23397-23407.

[19] D.L. Jarvis, Baculovirus-insect cell expression systems, Methods Enzymol. 463 (2009) 191-222.

[20] D.M. Kanter, D.L. Kaplan, Sld2 binds to origin single-stranded DNA and stimulates DNA annealing, Nucleic Acids Res. 39 (2011) 2580-2592. 